\title{
SEMIMAGNETIC SEMICONDUCTORS
}

\author{
J.A. GAJ \\ Institute of Experimental Physics, Warsaw University \\ Hoża 69, 00-681 Warszawa, Poland
}

\begin{abstract}
This paper contains a description and explanation of selected basic phenomena characteristic of bulk semimagnetic semiconductors: giant Faraday rotation and giant Zeeman splitting, mean field approximation and its limitations, ion-ion interaction, its measurements and its influence on magnetic properties, etc. The basic part is followed by a discussion of a personal choice of new perspectives open by the introduction of diluted magnetic semiconductors in semiconductor quantum structures. In particular, the role of magnetic ions in characterization of semiconductor quantum structures and low-dimensional excitonic systems is analyzed. Particular attention is paid to magnetooptical phenomena and magnetooptical experimental methods in large gap II-VI semimagnetic semiconductors.
\end{abstract}

PACS numbers: 75.50.Pp, 78.20.Ls, 71.70.Gm, 75.30.Et

\section{Basics (in bulk crystals)}

\subsection{Introduction}

Semimagnetic semiconductors, known also as diluted magnetic semiconductors (DMS), have been present in the landscape of semiconductor physics since late seventies. Extensive information on this class of materials can be found in successively published review papers [1], books [2,3], and book chapters $[4,5]$. Limited size of this paper imposed a narrowing of the area covered: a number of important phenomena, such as magnetic polarons, transport properties, specific properties of III-V DMS, and many others, are not discussed here.

\subsubsection{Idea of J.K. Furdyna}

Studies of diluted magnetic semiconductors owe their inspiration to J.K. Furdyna (Purdue University). Being an expert in microwave studies of solids, he wanted to apply his methods to semiconductors with large concentration of free carriers (such as zero-gap $\mathrm{HgTe}$ ) containing magnetic ions (e.g., $\mathrm{Mn}^{++}$). In principle the presence of the free carrier plasma prevents penetration of microwaves into such materials. Furdyna planned to use a transmission window below the plasma frequency, opened by magnetic field for helical microwave modes. 


\subsubsection{Growth of first DMS crystals}

Warsaw semiconductor physics community was well suited to undertake development of DMS studies. Besides the semiconductor crystal growth facilities present in Warsaw since early sixties, there was a sustained interest in transition metal impurities, introduced and maintained at Warsaw University by Jacek Baranowski. A group led by K. Leibler developed a well equipped laboratory of electron spin resonance at the Institute of Physics of Polish Academy of Sciences. The first attempt to grow alloys suggested by Jacek Furdyna was done by Witold Giriat at the Institute of Physics of Polish Academy of Sciences. At the beginning Mn was incorporated as an impurity [6], then introduced in larger quantities, typical of mixed crystals [7]. However, the works of that early period did not stimulate any larger interest, since the unique features of semimagnetic semiconductors were not revealed. A next attempt to attract a wide attention to this class of semiconductor compounds was undertaken by Robert Gałazka, also at the Institute of Physics of Polish Academy of Sciences. This time the attempt was fully successful and made of Robert Gałazka the acknowledged initiator of DMS studies. Very soon the works done on the crystals he produced and studied attracted a wide interest for this new class of semiconducting compounds with magnetic properties.

\subsubsection{Definition of diluted magnetic semiconductors. Basic interactions}

By a diluted magnetic or semimagnetic semiconductor we shall understand a well known (e.g., II-VI) semiconductor with a part of cations replaced randomly by ions (e.g., $\mathrm{Mn}^{++}$) possessing a large magnetic moment.

The host semiconductor (CdTe, HgTe, or GaAs) should be well known. We introduce then the magnetic ions into a physical system of known characteristics and, as a consequence, we are able to describe the properties of the obtained alloy with a better precision. Looking for a good description we can start from a relatively simple problem of a single ion in the known host crystal and then gradually complicate the system, introducing interactions between the ions. Typical magnetic ions may be those of either transition- or rare-earth elements.

We distinguish two most important interactions in DMS:

- ion-carrier ( $s p-d$ for transition metal ions) interaction, usually described by a Heisenberg Hamiltonian

$$
\widehat{H}=-\sum_{i} J(r-\boldsymbol{R}) \boldsymbol{\sigma} \cdot \boldsymbol{S}_{i}
$$

where $J\left(\boldsymbol{r}-\boldsymbol{R}_{i}\right)$ represents a short-range operator acting on the orbital part of the carrier wave function while $\boldsymbol{\sigma}$ and $\boldsymbol{S}_{i}$ denote spin operators of the carrier and $i$-th magnetic ion respectively. This form has been first applied to semimagnetic semiconductors by Kossut [8].

- ion-ion interaction described also by a Heisenberg-type Hamiltonian in a natural notation

$$
\widehat{H}=-J_{i j} S_{i} \cdot S_{j} \text {. }
$$

The ion-ion interaction in DMS is indirect and originates mainly from the ioncarrier interaction.

In what follows we shall consider various consequences of these two interactions for the properties of semimagnetic semiconductors. 


\subsubsection{Examples of semimagnetic semiconductors, first experiments}

$\mathrm{Hg}_{1-x} \mathrm{Mn}_{x} \mathrm{Te}$ - a narrow gap material, grown in the form of bulk crystals for Mn mole fraction $x \leq 0.3 . \mathrm{Hg}_{1-x} \mathrm{Mn}_{x}$ Te crystals from Warsaw were studied in the laboratory of C. Rigaux (Ecole Normale Supérieure in Paris) by means of infrared magnetooptical measurements. Giant spin splittings of band electron states were revealed and linked to ion-carrier exchange interaction [9]. Among phenomena characteristic of this material, oscillatory variation with temperature of Shubnikov-de Haas effect was reported [10].

$\mathrm{Cd}_{1-x} \mathrm{Mn}_{x} \mathrm{Te}$ - a wide energy gap from $1.6 \mathrm{eV}$ for $x=0$ to $3.5 \mathrm{eV}$ for $x=1$. Characteristic effects include giant Zeeman splitting of excitonic states and the resulting giant Faraday rotation. Studies of $\mathrm{Cd}_{1-x} \mathrm{Mn}_{x} \mathrm{Te}$ crystals have been initiated at Kiev [11] and independently at Warsaw [12]. Giant Faraday rotation was reported and linked to excitonic Zeeman splittings.

\subsection{Ion-ion (d-d) interaction}

\subsubsection{Basic ideas}

As mentioned in 1.1.4, the interaction between two magnetic ions can be expressed to a good approximation in the Heisenberg form (Eq. (2)).

In most semimagnetic semiconductors this interaction is known to be of antiferromagnetic $(\mathrm{AF}) \operatorname{sign}\left(J_{i j}<0\right)$ and of a short range. Various methods have been used to determine its strength. It is convenient to rewrite the Hamiltonian of the interaction in terms of squares of operators of the total spin $S$ of the pair and of individual spins of each ion

$$
\begin{aligned}
\widehat{H}= & -J_{i j} S_{i} \cdot S_{j}=-\frac{1}{2} J_{i j}\left[\left(S_{i}+S_{j}\right)^{2}-S_{i}^{2}-S_{j}^{2}\right] \\
& =-\frac{1}{2} J_{i j}\left[S(S+1)-S_{i}\left(S_{i}+1\right)-S_{j}\left(S_{j}+1\right)\right] .
\end{aligned}
$$

If we consider the example of $\mathrm{Mn}^{++}$spins, we get $S_{i}=S_{i}=5 / 2$ and $\widehat{H}=$ $-\frac{1}{2} J_{i j} S(S+1)+$ const. This means that for a negative (antiferromagnetic) value of the ion-ion exchange constant, we have a nonmagnetic $S=0$ ground state and then five excited states separated from each other by uniformly increasing energetic distances $E_{S}-E_{S-1}=-S J_{i j}$. An external magnetic field will split each state in $2 S+1$ components, as represented in Fig. 1.

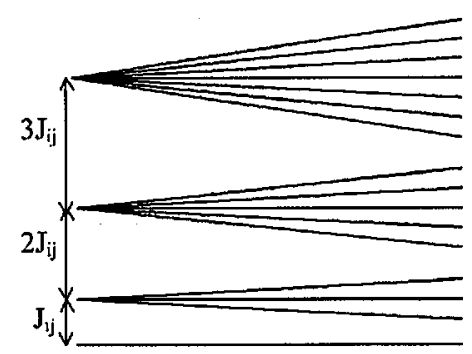

Fig. 1. Scheme of the ground state of a $\mathrm{Mn}^{++}$ion pair and of three of its excited states in a magnetic field. 
The ion-ion interaction depends on distance, which in case of a substitutional alloy can assume discrete values determined by the crystal structure. In the following we shall denote the nearest neighbor interaction constant $J_{1}$, the next-nearest neighbor one $-J_{2}$, and so on. Among many existing methods of determination of these values, here are some useful ones.

\subsubsection{Determination of ion-ion interaction constants}

1.2.2.1. The Curie-Weiss law. Reliable but not very detailed information on the ion-ion interaction is supplied by high-temperature susceptibility. If the temperature is high enough, the susceptibility obeys usually the Curie-Weiss law, which for the simplest spin-only case can be written as

$$
\chi(T, x)=\frac{C(x)}{T-\Theta(x)},
$$

where the Curie constant

$$
C(x)=x \frac{\left(g \mu_{\mathrm{B}}\right)^{2} S(S+1)}{3 k_{\mathrm{B}}} \frac{N}{V}
$$

and the Curie-Weiss temperature

$$
\Theta=\frac{1}{3 k} x S(S+1) \sum_{i} J_{i} n_{i}
$$

are proportional to the magnetic ion mole fraction $x$ and depend on the total spin $S$ of an ion, interaction constants $J_{i}$ with $i$-th neighbors and numbers $n_{i}$ of cations in respective coordination spheres [13]. From the Curie-Weiss temperature we know the sum of ion-ion exchange integrals, weighted by the numbers of cations in respective coordination spheres. However, since the ion-ion interaction is of a short range, in a first approximation we can neglect the interaction with all the neighbors but the nearest ones. For example, the Curie-Weiss temperature of CdMnTe, measured by Spałek et al. [13], equals $\Theta=-470 K x$. Attributing it to the nearest neighbor interaction only, we obtain $J_{1} / k_{\mathrm{B}}=-13.4 \mathrm{~K}$, not too far from values obtained from more precise methods. If we know precisely $J_{1}$, we can use the Curie-Weiss temperature to estimate $J_{2}$, but such a method is less reliable. 1.2.2.2. Magnetization steps. A very elegant method of studying ion-ion interaction uses high-field magnetization measurements.

As visible in Fig. 2, at certain magnetic field values the slope of the ground state energy of the pair versus magnetic field changes. Since magnetization corresponds to the derivative of the energy with regard to magnetic field, at sufficiently low temperature a step-like magnetization will be observed. Experiments have shown [14] that for mole fraction values around 5\%, steps of magnetization due to nearest neighbor pairs can indeed be observed at temperatures of order of $1 \mathrm{~K}$. The first step should occur at magnetic field $B=2 J / g \mu_{\mathrm{B}}$. A more detailed analysis predicts a shift of the field at which steps occur due to interaction with more distant neighbors. The height of the steps provides information on the number of pairs. In case of CdMnTe the data confirm random distribution of $\mathrm{Mn}^{++}$ions.

1.2.2:3. Inelastic neutron scattering. Neutrons can excite magnetic ion pairs to the excited states separated in energy by multiples of the interaction constant, which 


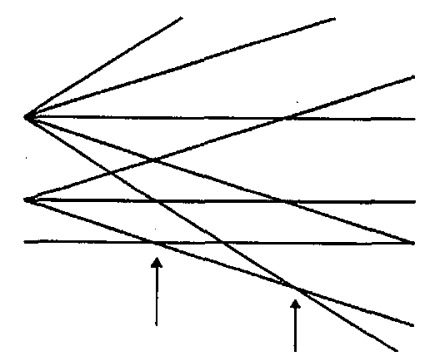

Fig. 2. A schematic diagram of three lowest-lying levels of an Mn pair and of their splitting in magnetic field.

can be therefore determined directly from energy spectra of diffracted neutrons. Such measurements have been done for a number of semimagnetic semiconductors [15].

\subsubsection{Ion-ion interaction in various materials}

Numerical values of ion-ion exchange constants can be found elsewhere [5]. It is risky to conclude on trends from data obtained in different experiments, since considerable scatter of the data occurs. Nevertheless, de Jonge and Swagten [16] find that a universal dependence of ion-ion exchange constant on inter-ion spacing can describe the properties of several semimagnetic compounds with Mn ions. A fast decay of the interaction strength with distance is found (a power law with an exponent of -6.8 ).

\subsubsection{Low-temperature magnetization}

Ion-ion interaction constants and the distribution of magnetic ions over the cation sublattice determine magnetic properties of the material. Numerous studies have been performed to determine the phase diagrams of semimagnetic semiconductors, containing typically paramagnetic, antiferromagnetic, and spin-glass phases. From the practical point of view low temperature (pumped $\mathrm{He}$ ) magnetization of semimagnetic semiconductors in magnetic fields up to a few tesla presents a special interest, since it determines most of magnetooptical properties at typical experimental conditions. Magnetization data is often described in terms of a modified Brillouin function. The magnetization per, e.g., Mn ion expressed in terms of its spin component along the magnetic field reads then

$$
\left\langle S_{z}\right\rangle=-S_{0} B_{S}\left[\frac{g \mu_{\mathrm{B}} S B}{k_{\mathrm{B}}\left(T-T_{0}\right)}\right],
$$

where $S=5 / 2$ is Mn spin value, $g=2$ is Mn gyromagnetic factor, $\mu_{\mathrm{B}}, k_{\mathrm{B}}$, and $T$ are the Bohr magneton, the Boltzmann constant, and temperature respectively;

$$
B_{S}(\xi)=\frac{2 S+1}{2 S} \operatorname{cth}\left(\frac{2 S+1}{2 S} \xi\right)-\frac{1}{2 S} \operatorname{cth}\left(\frac{1}{2 S} \xi\right)
$$

is the Brillouin function. The two empirical parameters: effective spin saturation value $S_{0}$ and a temperature correction $T_{0}$ depend on $\mathrm{Mn}$ mole fraction of the alloy and were introduced to fit the experimental data.

The analysis in case of dilute ( $x$ up to a few \%) alloys can be performed in terms of two classes of ions: those with no nearest neighbors and those forming 
nearest neighbor pairs. If the magnetic ion distribution is not too far from random, we can neglect bigger clusters. In a typical $\left(J_{i j}<0\right)$ semimagnetic semiconductor, at relatively low magnetic fields the pairs will be in their ground $(S=0)$ state and will not contribute to magnetization. The effective saturation value $S_{0}$ will be smaller than the spin $S_{i}$ of a single ion, expressing the exclusion of pairs from the magnetization. The $S_{0}$ value carries therefore information on the spatial distribution of the ions. If the ions having no nearest neighbors were completely isolated, the magnetization would follow a standard Brillouin function $\left(T_{0}=0\right)$. The non-zero value of $T_{0}$ expresses an influence of more distant neighbors. Being relatively small, this influence can be described in terms of a mean field approximation, producing a $T_{0}$ proportional to the magnetic ion mole fraction $x$. The applicability of such an analysis can be estimated from Table containing data for CdMnTe. For more concentrated alloys, the magnetization is strongly suppressed by ion-ion interaction.

\section{TABLE}

Brillouin function parameters of CdMnTe magnetization, after Gaj et al. [21]. Theoretical estimate of $S_{0}$ given for a ran'dom distribution.

\begin{tabular}{c|c|c|c}
\hline \hline$x$ & \multicolumn{2}{|c|}{$S_{0}$} & \multicolumn{1}{c}{$T_{0}$} \\
\cline { 2 - 3 } & exp. & theor. & {$[\mathrm{K}]$} \\
\hline 0.005 & 2.11 & 2.35 & -0.29 \\
0.01 & 2.12 & 2.22 & -0.68 \\
0.02 & 1.97 & 1.96 & -0.94 \\
0.05 & 1.54 & 1.35 & -2.29
\end{tabular}

\subsection{Influence of magnetic ions on charge carriers}

\subsubsection{Giant Faraday effect and its origin}

1.3.1.1. Basic ideas. By the Faraday effect we understand rotation of polarization plane of light propagating along a magnetic field (the Faraday configuration). The rotation angle $\vartheta$ is twice smaller than the phase shift between the two circular components of the light transmitted by the sample

$$
\vartheta(\omega)=\frac{\omega}{2 c} d\left[n_{+}(\omega)-n_{-}(\omega)\right] .
$$

Usually we introduce the Verdet constant, i.e., rotation angle per unit thickness of the sample

$$
\vartheta(\omega) / d=\frac{\omega}{2 c}\left[n_{+}(\omega)-n_{-}(\omega)\right]
$$

1.3.1.2. Description of experimental Faraday rotation spectra. From the very beginning of studies of semimagnetic semiconductors, giant values of the Faraday 
rotation were reported in large gap DMS. The authors searched for an adequate description of the Faraday rotation spectra. The first efforts were centered on a search for the type of singularity at the transition energy [12]. An improved model based on calculations of spectral moments was shown to describe the Faraday rotation of CdMnTe with a better accuracy [17]. The best currently available description of the refractive index due to interband transitions is due to Tanguy [18]. His models can certainly provide a precise description of interband Faraday rotation.

\subsubsection{Mean field approximation}

While describing the influence of the carrier-ion interaction on the properties of a large-gap semimagnetic semiconductor it is important to realize the relative numbers: we have usually to do with a number of magnetic ions comparable to the total number of atoms in the crystal, while the number of carriers is smaller by orders of magnitude; the material is often semi-insulating and the only carriers are those created by light. Therefore a carrier interacts usually with a great number of magnetic ions. This inequality justifies the most commonly used approach: an external magnetic field aligns the magnetic ions, which in turn act on carriers via ion-carrier interaction. This action has been described successfully in the mean field approximation, where the spin operators of magnetic ions have been replaced by their thermal average. Furthermore, the mean field approximation is usually completed by the virtual crystal approximation, restoring crystal periodicity: the random distribution of magnetic ions and host cations over the cation sublattice is replaced by a periodical structure with artificial cations possessing properties of the host cation and the magnetic ion, averaged using occupation probabilities as weights. Within these approximations the carrier-ion Hamiltonian (Eq. (1)) becomes $\widehat{H}=-N_{0} \alpha x \sigma_{z}\left\langle S_{z}\right\rangle$ for the conduction electrons and $\widehat{H}=-N_{0} \beta x \sigma_{z}\left\langle S_{z}\right\rangle$ for the valence-band ones. This form of the Hamiltonian applied to the states at $\Gamma$-point of the Brillouin zone produces a splitting of the conduction band in two components of opposite spins by $\Delta E_{\mathrm{c}}=\left|N_{0} \alpha x\left\langle S_{z}\right\rangle\right|$ and a splitting of the valence band in four equidistant components with the external ones split by $\Delta E_{\nu}=\left|N_{0} \beta x\left\langle S_{z}\right\rangle\right|$. In order to obtain a non-vanishing $\left\langle S_{z}\right\rangle$ value one has to apply a magnetic field. Acting on the conduction- and valence-band states, this field will modify the wave functions of the carriers, produce the Landau quantization, usual spin splittings, etc. These effects are of primary importance in narrow gap materials, but in large-gap semiconductors they may often be neglected compared to the giant spin splitting due to ion-carrier interaction. In such an approximation we can still apply the notion of the wave vector and consider unperturbed bands except of the exchange splittings.

In case of $\mathrm{Mn}^{++}$ions possessing no orbital magnetic moment, the thermal average $\left\langle S_{z}\right\rangle$ represents the magnetization per magnetic ion and $x\left\langle S_{z}\right\rangle$ magnetization per cation. Considering non-vanishing wave vectors within the above approximations, Gaj et al. [19] obtained a fairly complicated band structure as a result of combined carrier-ion exchange- and $k \cdot p$ interactions. 
1.3.3. Excitonic Zeeman effect, determination of ion-carrier (sp-d) exchange integrals in $C d M n T e$

Magnetoreflectivity measurements on CdMnTe at low temperatures revealed that the $1 S$ excitonic ground state transition splits into six components: two of them visible in each circular polarization in the Faraday configuration (light propagating along magnetic field) and additional two visible in $\pi$ polarization $(\boldsymbol{E} \| \boldsymbol{B})$ in the Voigt configuration (light propagation perpendicular to the magnetic field). The scheme of the splittings and selection rules are shown in Fig. 3.
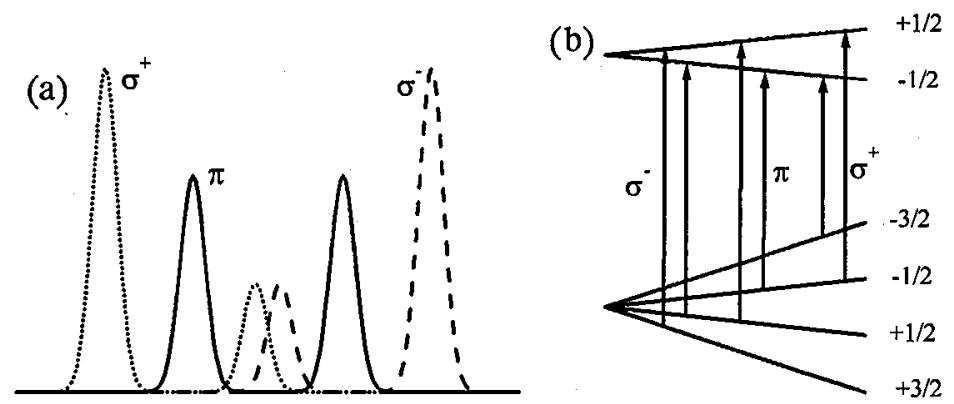

Fig. 3. Splitting scheme (a) and selection rules (b) in magnetic field for $1 S$ excitonic transition in CdMnTe.

All the splittings have been shown to be proportional to magnetization [20]. This fact confirms the applicability of the mean field approximation not only to free carrier states but also to the excitonic ones. This means that we can associate an exciton with each pair of exchange-split components of energy bands. Such a result is obtained by neglecting electron-hole exchange interaction which would couple excitonic states associated with various pairs of the subbands. In fact, electron-hole exchange interaction energy is below $1 \mathrm{meV}$, very small compared to giant Zeeman splittings, which at liquid He temperatures and a few tesla are typically of order of $100 \mathrm{meV}$. Assuming that the excitonic effects are identical for all the subbands, relative transition intensities can be calculated for simple interband transitions, assuming conduction wave functions in the form

$$
|S\rangle \uparrow, \quad|S\rangle \downarrow
$$

and the valence ones as

and

$$
\frac{1}{\sqrt{6}}(|X+\mathrm{i} Y\rangle \downarrow-2|Z\rangle \uparrow), \quad \frac{1}{\sqrt{6}}(|X-\mathrm{i} Y\rangle \uparrow+2|Z\rangle \downarrow)
$$

$$
\frac{1}{\sqrt{2}}|X+\mathrm{i} Y\rangle \uparrow, \quad \frac{1}{\sqrt{2}}|X-\mathrm{i} Y\rangle \downarrow
$$

for light- and heavy-hole bands, respectively. Six non-vanishing transition prob- . abilities are then obtained as squares of absolute values of electric dipole matrix elements between the corresponding states. In particular, in the Faraday configuration the heavy-hole transitions are three times stronger than the light-hole ones. Experimental spectra confirm qualitatively the calculated selection rules. 
By plotting excitonic Zeeman splitting versus magnetization one obtains straight lines with slope determined by exchange integrals, e.g., for the "strong" components observed in the Faraday configuration the Zeeman splitting in the mean field approximation (Sec. 1.3.2) is

$$
\Delta E_{\mathrm{e}}=E_{-}-E_{+}=N_{0}(\beta-\alpha) x\left\langle S_{z}\right\rangle,
$$

where indices - and + denote circular polarizations (please note that $\left\langle S_{z}\right\rangle$ is negative). For the "weak" components the splitting is

$$
\Delta E_{\mathrm{e}}=E_{-}-E_{+}=N_{0}(\beta / 3+\alpha) x\left\langle S_{z}\right\rangle .
$$

Such plots have been shown to be common for various Mn mole fraction values and supply reliable values of exchange integrals. In CdMnTe commonly accepted values are $N_{0} \alpha=0.22 \mathrm{eV}$ and $N_{0} \beta=-0.88 \mathrm{eV}$ for the conduction- and valence band respectively.

The procedure described above has been applied to many other semimagnetic semiconductors resulting in determination of the $s p-d$ exchange constants. It is interesting to note that the values of valence band $p-d$ constant in $\mathrm{Cr}$ containing alloys are positive [21], as opposed to most DMS materials. A very interesting case occurs for Mn-containing III-V compounds [1], where a strong influence of the free carriers complicates the analysis.

\subsubsection{Zeeman effect for excitons above the fundamental energy gap}

Twardowski et al. [22] reported such studies for excitons in CdMnTe involving holes from the spin-orbit split-off valence band at energies about $2.5 \mathrm{eV}$ $\left(E_{0}+\Delta_{0}\right.$ in Fig. 4). The measured Zeeman splittings were described in terms of the same $s p-d$ interaction constants as for the transitions at the fundamental energy gap. These measurements allowed the authors to determine also a precise value of the spin-orbit splitting of the CdTe valence band, equal to $0.948 \mathrm{eV}$.

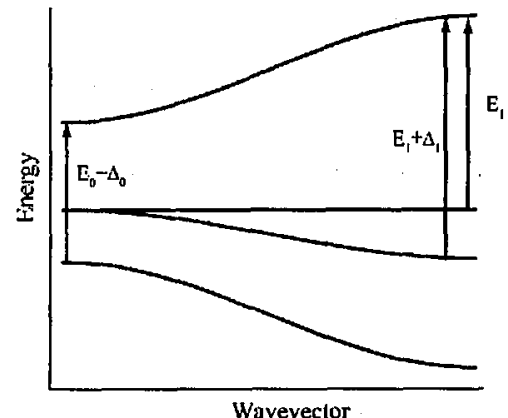

Fig. 4. A scheme of optical transitions in the Brillouin zone of CdMnTe.

Zeeman effect was also studied for transitions at the $L$-point of the Brillouin zone. These studies were initiated by E. Dudziak et al. in 1982. The first quantitative results have been obtained on CdMnTe by Ginter et al. [23]; Coquillat et al. [24] have performed measurements for $\mathrm{ZnMnTe}$ and $\mathrm{HgMnTe}$. In all cases the measured splitting was much smaller than the width of the reflectivity structures. Therefore only some kind of averaged splitting values was possible to obtain 
by polarization modulation techniques. The splitting values of the $E_{1}$ transition were considerably smaller than those measured at the center of the Brillouin zone. An attempt by Ginter et al. [23] to compute the Zeeman splittings at the $L$-point in terms of a tight binding model predicted two reasons for the observed reduction: reduction of splittings of the energy bands and relaxation of selection rules, both due to mixing between the conduction- and valence-band wave functions. However, they calculated a reduction smaller than that measured experimentally. To explain the difference, a $k$-dependence of the $p-d$ exchange integral was postulated. Besides, the calculations predicted for $E_{1}$ and $E_{1}+\Delta_{1}$ transitions (involving two valence-band components split by spin-orbit interaction) splittings identical in absolute value and opposite in sign. This result has been confirmed by experimental data of Coquillat et al. [24] and later by a more realistic calculation performed by Bhattachargee [25]. Dependence of $s p-d$ interaction on wave vector in quantum wells has been also reported by Mackh et al. [26] based on the Zeeman effect results in short-period CdMnTe/CdMgMnTe superlattices. The confinement-induced reduction of giant Zeeman splittings was analyzed theoretically by Bhattachargee [27], who explained qualitatively the results of Mackh et al. [26] and predicted much stronger effects to be expected in quantum dots.

\subsubsection{Influence of magnetic fluctuations on band electron states}

Magnetic fluctuations can mix effective mass states of different $k$ vector values [28]. This phenomenon cannot be described within the mean field approximation. In a second-order perturbation calculation, the influence of fluctuations summed over all the $k$ states from a band will lower the conduction band minimum (shift up the valence band top) by a value

$$
\Delta E(k, T)=\sum_{q} \frac{\frac{1}{3} j(j+1)\left|J_{k q}\right|^{2} \Gamma_{q}}{E_{0}(k)-E_{0}(k+q)},
$$

where $J_{k q}=\int \mathrm{d}_{3} r u_{k+q}^{*}(\boldsymbol{r}) u_{k}(\boldsymbol{r}) J(r)$ and $\Gamma_{q}=N^{-1} x \sum_{j}\left\langle\boldsymbol{S}_{0} \cdot \boldsymbol{S}_{j}\right\rangle_{T} \exp \left(\mathrm{i} \boldsymbol{q} \cdot \boldsymbol{R}_{j}\right)$.

The coefficient $\Gamma_{q}$ describing magnetic fluctuations was approximated by its long-wavelength limit $\Gamma_{0}$ and through dissipation-fluctuation theorem related to the product of magnetic susceptibility and temperature. A good description of energy gap variation with temperature was obtained for several DMS compounds. The only free parameter of the model - an integral of the square of the exchange constant $J_{k q}$ over the Brillouin zone was determined from fits of experimental temperature dependence of the energy gap. The same approach allowed Bylsma et al. [29] to explain the dependence of the energy gap of ZnMnSe not only on temperature but also on composition (strongly nonlinear).

\section{Quantum structures of semimagnetic semiconductors}

\subsection{Mean field approximation for quantum structures}

Introduction of magnetic ions in semiconductor heterostructures opens a wealth of new possibilities related to giant energy shifts in magnetic field caused by the exchange interaction. To name a few examples, spin superlattices [30], observation of above-barrier states [31], type 1 to type 2 transition [32], easily 
tunable resonant tunneling [33], and many other effects can be created or facilitated by using semimagnetic semiconductors.

\subsubsection{Band offsets}

Unlike bulk crystals, for heterostructures we are interested not only in variation of energy gap as a function of alloy composition, but also in the distribution of this variation between the conduction- and the valence band. A commonly used parameter, the relative valence band offset

$$
\text { vbo }=-\frac{E_{\mathrm{v}}(x)-E_{\mathrm{v}}(0)}{E_{\mathrm{g}}(x)-E_{\mathrm{g}}(0)}
$$

determines what fraction of the band gap variation occurs in the valence band. With the above choice of the sign we obtain positive (and smaller than 1) vbo for type 1 heterostructures, where both carriers are confined in the same layer.

Similarly as in the case of classical III-V GaAs/GaAlAs quantum wells, commonly accepted values of relative band offsets have not been determined immediately. In early stages of studies of semimagnetic heterostructures the band offsets were determined primarily from the Zeeman effect measurements. For $\mathrm{CdMnTe} / \mathrm{CdTe}$ system the published values of vbo ranged from almost zero [34] to $46 \%$ [35] depending on the Mn mole fraction of CdMnTe crystals and on the method of determination. Only when a method unrelated to Zeeman effect has been used by Kuhn-Heinrich et al. [36], a composition-independent value of vbo $=0.30$ has been obtained. At present the most commonly used values are between 0.30 and 0.40 .

\subsubsection{Interface characterization}

The problem with using the Zeeman effect as a tool to determine the band offsets originates in interface effects. If the interface between, say, a CdTe quantum well and a CdMnTe barrier is not perfectly abrupt but contains an intermixed diluted region, the Zeeman effect of an exciton confined in the quantum well may be primarily influenced by the ions in that region because the dilution allows them to align easier in an external magnetic field (see Sec. 1.2.4). The enhancement of the Zeeman splitting due to intermixing may be as high as an order of magnitude. To compensate for the dilution effect at interfaces, authors of early publications modified the band offsets. In case of excitonic Zeeman effect a decrease in the vbo was helpful since it made the hole penetrate deeper in the barriers, and the hole splitting, being in the bulk four times stronger than that of the electron, made the total excitonic Zeeman splitting bigger. In case of electron spin-flip energy data, it was necessary to decrease the vbo to imitate the enhancement of conduction electron splitting due to dilution at interfaces.

This sensitivity, annoying from the point of view of band offsets determination, allowed to create an interface characterization method [37] known as spin tracing. By fitting the experimental Zeeman splitting of excitons confined in $\mathrm{CdTe} / \mathrm{CdMnTe}$ quantum wells, the width of the interface profile can be determined. Spin tracing can be used to study, e.g., interdiffusion due to annealing [38] or the influence of growth temperature on interface width. 


\subsection{Fluctuations in quantum structures}

\subsubsection{Influence of magnetic fluctuations on band offsets in heterostructures}

The influence of fluctuations discussed in Sec. 1.3.5 is much stronger for the valence- than for the conduction band due to the difference in effective masses and $s p-d$ exchange integrals. Therefore a variation of temperature can lead to a change of the valence band offset in $\mathrm{CdTe} / \mathrm{CdMnTe}$ heterostructures. Such an effect has been verified experimentally by Kossacki et al. [39], who found a significant variation of vbo with temperature in agreement with the fluctuation model.

2.2.2. Dependence of effective values of ion-carrier exchange integrals on temperature

Magnetic fluctuations can also lead to a localization of excitonic states. The localization can in turn modify the wave functions and cause relaxation of polarization selection rules, leading to a decrease in effectively observed Zeeman splittings. This decrease can be expressed in the form of effective ion-carrier exchange integral value decreasing with temperature. Such an effect has been reported by Kossacki et al. [40] in CdTe/CdMnTe quantum wells and superlattices.

\subsection{Charged excitons in semimagnetic semiconductors}

A charged exciton or trion consists of three carriers, i.e., two electrons and a hole or two holes and an electron. In bulk crystals these complexes are too weakly bound to be observed. A confining potential of a quantum well brings the carriers closer together, increasing the binding energy and thus making trions observable. The first experimental observation of negatively charged excitons $\mathrm{X}^{-}$was done by Kheng et al. [41] in modulation-doped $\mathrm{CdTe} / \mathrm{CdZnTe}$ multiple quantum wells. This observation was rapidly followed by experimental evidence for both kinds of excitons in III-V and II-VI $2 \mathrm{D}$ systems.

In a magnetic field the trion absorption line, unlike that of an exciton in an isolating crystal, exhibits a net circular polarization resulting from the selection rules and the Pauli exclusion principle in presence of spin-polarised free carrier gas. Introduction of semimagnetic semiconductors in such experiments added a new possibility of tuning the polarization of carrier gas by a small external magnetic field. The giant band splittings in magnetic field give us a tool for investigation of trions in the low-field range, where the exciton wave function is perturbed negligibly. Thus we obtain pure information about population effects $[42,43]$.

\subsection{Ferromagnetism in quantum structures}

Alignment of magnetic ions by carriers on the scale of a bulk crystal was studied in IV-VI compounds by Story et al. [44], who was able to explain quantitatively the observed ferromagnetism by the influence of free holes, possessing a large density of states. In classical II-VI semimagnetic compounds the necessary large density of carriers was more difficult to obtain*. Only in the late nineties the

*An important perspective related to ferromagnetism in DMS crystals has been opened by introduction of magnetic ions in III-V compounds - see H. Ohno, in Ref. [1]. 
first ferromagnetic transition in a modulation $p$-doped CdMnTe quantum well was reported [45]. The latter system has several interesting features. As predicted by Dietl et al. [46], the Curie temperature is almost independent of hole density, since in a 2D system the density of states is concentration-independent. Spontaneous magnetization, unlike in most ferromagnetics, is oriented perpendicularly to the $2 \mathrm{D}$ layer due to the anisotropy of confined holes.

\section{Acknowledgment}

This work has been partially supported by the Committee for Scientific Research (grant PBZ 28.11).

\section{References}

[1] H. Ohno, Science 281, 951 (1998) (III-V compounds); J.K. Furdyna, J. Appl. Phys. 64, R29 (1988); N.B. Brandt, V.V. Moschchalkov, Adv. Phys. 33, 193 (1984); J.A. Gaj, J. Phys. Soc. Jpn. 49, 797 (1980).

[2] Diluted Magnetic Semiconductors, Ed. M. Jain, World Scientific, Singapore 1991.

[3] Semiconductors and Semimetals, Vol. 25, Diluted Magnetic Semiconductors, Eds. J.K. Furdyna, J. Kossut, Academic Press, San Diego (CA) 1988.

[4] T. Dietl, in: Handbook of Semiconductors, Eds. T.S. Moss, S. Mahajan, Elsevier Sci. B.V., Amsterdam 1997, p. 1521.

[5] J. Kossut, W. Dobrowolski, in: Narrow-Gap II-VI Compounds for Optoelectronic and Electromagnetic Applications, Ed. P. Capper, Chapman \& Hall, London 1997, p. 401.

[6] K. Leibler, W. Giriat, Z. Wilamowski, R. Iwanowski, Phys. Status Solidi B 47, 405 (1971).

[7] J. Stankiewicz, W. Giriat, M.V. Bien, Phys. Status Solidi B 18, 485 (1975).

[8] J. Kossut, Phys. Status Solidi B 72, 359 (1975).

[9] G. Bastard, C. Rigaux, A. Mycielski, Phys. Status Solidi B 79, 585 (1977).

[10] M. Jaczyński, J. Kossut, R.R. Gałazka, Phys. Status Solidi B 88, 73 (1978).

[11] A.V. Komarov, S.M. Ryabchenko, O.V. Terletskii, I.I. Zheru, R.D. Ivanchuk, $Z h$. Eksp. Teor. Fiz. 73, 608 (1977).

[12] J.A. Gaj, R.R. Gałazka, M. Nawrocki, Solid State Commun. 25, 193 (1978), reprinted in Solid State Commun. 88, 923 (1993).

[13] J. Spałek, A. Lewicki, Z. Tarnawski, J.K. Furdyna, R.R. Gałazka, Z. Obuszko, Phys. Rev. B 33, 3407 (1986).

[14] S. Foner, Y. Shapira, D. Heiman, P. Becla, R. Kershow, K. Dwight, A. Wold, Phys. Rev. B 39, 11793 (1989).

[15] See, e.g., T. Giebułtowicz, T.M. Holden, in Ref. [3], p. 125.

[16] W.J.M. de Jonge, H.J.M. Swagten, J. Magn. Magn. Mater. 100, 322 (1991).

[17] J.A. Gaj, in Ref. [3], p. 296.

[18] C. Tanguy, Phys. Rev. Lett. 75, 4091 (1995).

[19] J.A. Gaj, J. Ginter, R.R. Gałązka, Phys. Status Solidi B 89, 655 (1978).

[20] J.A. Gaj, R. Planel, G. Fishman, Solid State Commun. 29, 435 (1979); reprinted in Solid State Commun. 88, 927 (1993). 
[21] W. Mac, A. Twardowski, M. Demianiuk, Phys. Rev. B 54, 5528 (1996).

[22] A. Twardowski, Solid State Commun. 36, 927 (1980).

[23] J. Ginter, J.A. Gaj, Le Si Dang, Solid State Commun. 48, 849 (1983).

[24] D. Coquillat, J.P. Lascaray, J.A. Gaj, J. Depportes, J.K. Furdyna, Phys. Rev. B 39, 10088 (1989).

[25] A.K. Bhattachargee, in: Proc. $X X$ Int. Conf on Physics of the Semiconductors, Thessaloniki 1990, Eds. E.M. Anastassakis, J.D. Joannopoulos, World Scientific, Singapore 1990, p. 763 .

[26] G. Mackh, W. Ossau, A. Waag, G. Landwehr, Phys. Rev. B 54, R5227 (1996).

[27] A.K. Bhattachargee, Phys. Rev. B 58, 15660 (1998).

[28] J.A. Gaj, A. Golnik, Acta Phys. Pol. A 71, 197 (1987).

[29] R.B. Bylsma, W.M. Becker, J. Kossut, U. Debska, D. Yoder-Short, Phys. Rev. B 33, 8207 (1986).

[30] W.C. Chou, A. Petrou, J. Warnock, B.T. Jonker, Phys. Rev. Lett. 67, 3820 (1991); N. Dai, H. Luo, F.C. Zhang, N. Samarth, M. Dobrowolska, J.K. Furdyna, Phys. Rev. Lett. 67, 3824 (1991).

[31] N. Dai, L.R. Ram-Mohan, H. Luo, G.L. Yang, F.C. Zhang, M. Dobrowolska, J.K. Furdyna, Phys. Rev. B 50, 18153 (1994).

[32] X. Li, A. Petrou, J. Warnock, B.T. Jonker, G.A. Prinz, J.J. Krebs, Phys. Rev. Lett. 63, 2280 (1989).

[33] I. Lawrence, S. Haacke, H. Mariette, W.W. Ruhle, H. Ulmer-Tuffigo, J. Cibert, G. Feuillet, Phys. Rev. Lett. 73, 2131 (1994).

[34] S.-K. Chang, A.V. Nurmmikko, J.-W.Wu, L.A. Kolodziejski, R.L. Gunshor, Phys. Rev. B 37, 1191 (1988).

[35] M.P. Halsall, D. Wolverson, J.J. Davies, Solid State Commun. 86, 15 (1993).

[36] B. Kuhn-Heinrich, W. Ossau, T. Litz, A. Waag, G. Landwehr, J. Appl. Phys. 75, 8046 (1994).

[37] J.A. Gaj, W. Grieshaber, C. Bodin-Deshayes, J. Cibert, G. Feuillet, Y. Merle d'Aubigné, A. Wasiela, Phys. Rev. B 50, 5512 (1994).

[38] P. Kossacki, Nguyen The Khoi, J.A. Gaj, G. Karczewski, T. Wojtowicz, E. Janik, A. Zakrzewski, M. Kutrowski, J. Kossut, Superlattices Microstruct. 16, 63 (1994).

[39] P. Kossacki, Nguyen The Khoi, A. Stachow, J.A. Gaj, G. Karczewski, J. Kossut, T. Wojtowicz, Nuovo Cimento D 17, 1537 (1995).

[40] P. Kossacki, Nguyen The Khoi, J.A. Gaj, G. Karczewski, J. Kossut, T. Wojtowicz, Phys. Rev. B 59, 7679 (1999).

[41] K. Kheng, R.T. Cox, Y. Merle d'Aubigné, Franah Bassani, K. Saminadayar, S. Tatarenko, Phys. Rev. Lett. 71, 1754 (1993).

[42] T. Wojtowicz, M. Kutrowski, G. Karczewski, J. Kossut, F.J. Teran, M. Potemski, Phys. Rev. B 59, R10437 (1999).

[43] P. Kossacki, Ph.D. thesis, Warsaw University 1998.

[44] T. Story, R.R. Gałązka, R.B. Frankel, P.A. Wolf, Phys. Rev. Lett. 56, 777 (1986).

[45] A. Haury, A. Wasiela, A. Arnoult, J. Cibert, S. Tatarenko, T. Dietl, Y. Merle d'Aubigné, Phys. Rev. Lett. 79, 511 (1997).

[46] T. Dietl, A. Haury, Y. Merle d'Aubigné, Phys. Rev. B 55, R3347 (1997). 